\title{
Use of weaning management group as a random effect for a more robust estimation of genetic parameters for post-weaning traits in Nellore cattle
}

\author{
V.B. Pedrosa ${ }^{1}$, J.P. Eler ${ }^{2}$, J.B.S. Ferraz ${ }^{2}$ and E. Groeneveld ${ }^{3}$ \\ ${ }^{1}$ Grupo de Melhoramento Animal, Departamento de Zootecnia, \\ Universidade Estadual de Ponta Grossa, Ponta Grossa, PR, Brasil \\ ${ }^{2}$ Grupo de Melhoramento Animal e Biotecnologia, \\ Departamento de Ciências Básicas, Universidade de São Paulo, Pirassununga, \\ SP, Brasil \\ ${ }^{3}$ Friedrich Loeffler Institute, Institute of Farm Animal Genetics, \\ Department of Animal Breeding and Genetic Resources, Neustadt, Germany \\ Corresponding author: V.B. Pedrosa \\ E-mail: vbpedrosa@uepg.br
}

Genet. Mol. Res. 13 (3): 7013-7021 (2014)

Received May 17, 2013

Accepted September 28, 2013

Published February 21, 2014

DOI http://dx.doi.org/10.4238/2014.February.21.7

\begin{abstract}
Data from 69,525 animals were used to compare two types of analyses, one of them having the weaning management group (WEMANG) included as an effect in the contemporary group ( $\mathrm{F}_{-}$ WEMANG) and the other considering the weaning management group as a random effect, not related to the mathematical model (R_WEMANG) for post-weaning traits. The components of (co)variance were estimated for pre-weaning traits (birth weight and weaning weight) and for postweaning traits [scrotal circumference (SC), weight gain from weaning to 18 months of age (WG) and muscle score (MUSC)] in Nellore cattle, based on a complete animal model. Heritability of SC, WG and MUSC for the F_WEMANG model was equal to $0.46 \pm 0.02,0.38 \pm 0.03$ and
\end{abstract}


$0.26 \pm 0.01$, and for the R_WEMANG model it was $0.45 \pm 0.02,0.31 \pm$ 0.03 and $0.25 \pm 0.01$, respectively. Genetic correlations between all the studied traits varied between $0.07 \pm 0.01$ and $0.77 \pm 0.03$ in F_WEMANG and between $0.02 \pm 0.01$ and $0.76 \pm 0.04$ in R_WEMANG. The R_ WEMANG model allowed a decrease in the number of contemporary groups as well as an increase in the number of observations per group without significant alterations in heritability coefficients, for the postweaning traits. Consequently, the analysis became more robust and avoided having contemporary groups with low variability.

Key words: Genetic correlation; Beef cattle; Contemporary group; Heritability

\section{INTRODUCTION}

During the last decades, some studies have attempted to obtain an operational model for estimation of variance components closer to the "true" model, that is, a model that better describes the real situation of data. Most of these studies were concerned with the genetic effects considered in the model and with the algorithms to solve the system of equations generated for prediction of genetic parameters (Ugarte et al., 1992).

Genetic evaluation models include contemporary groups (CG) to buffer the effects of variation due to the different environments in which the animals are maintained in each phase of their growth. For genetic evaluation of beef cattle, normally the mathematical models consider the management groups in different stages of their development in the concatenation of information to compose contemporary groups.

Due to managing policies, it is possible for breeders to wean animals from three or more different weaning management groups and to cluster them into different postweaning management groups, not necessarily using all the animals from one given weaning management group to create the post-weaning management group. Those groups are usually concatenated among them and with other fixed effects to form the CGs. The post-weaning CG is created by concatenating fixed effects of the weaning phase and fixed effects of the post-weaning phase. When effects are concatenated to create CGs of a given post-weaning trait, many CGs are formed with a small number of observations in each group.

Contemporary groups are considered fixed effects in the model and, so being, they need a sufficient number of observations for the solution of the system and for a robust comparison of animals between groups. Solutions for those small groups are neither reliable nor meaningful, due to the low levels or even lack of variability. Some authors choose to exclude these groups without even discussing the consequences of such loss of information for genetic evaluation (Vasconcelos et al., 2008). However, when CG effects are treated as random, the effective number of offspring of each animal that is being evaluated increases, and subsequently, prediction error variance decreases (Ugarte et al., 1992).

Accuracy in the prediction of genetic values is essential to the selection process (Pollak and Quaas, 1983). Genetic value is the function of additive genetic variance, and pre- 
diction accuracy depends on the variance of the prediction error, which greatly depends on the number of observations through which each animal contributes to the evaluation and the formation of the CGs. Therefore, eliminating CGs with few observations may generate biased genetic predictions (Henderson, 1975). Including the WEMANG as a random effect in the model and excluding the WEMANG from the CG allows a considerable decrease in the number of CGs with a small amount of observations. Thus, the data will be adjusted appropriately and the post-weaning contemporary groups will be more consistent.

The objective of this study was to assess the use of the WEMANG as a random effect in the estimation of genetic parameters for post-weaning traits in Nellore cattle, comparing variance components and genetic parameter estimates between the two methods.

\section{MATERIAL AND METHODS}

The animal records were obtained from three herds owned by Agropecuária CFM Ltda. [Agricultural Livestock CFM Ltd.]. Analyses were carried out by the Animal Breeding and Biotechnology Group of the Animal Science and Food Engineering College, University of São Paulo, Brazil (GMAB - FZEA/USP). The database contained records of Nellore born from 1984 until 2009 and the relationship matrix was composed of 165,539 records, including 61,249 males and 104,290 females. Only CGs with at least ten observations each and offspring from at least two different bulls were analyzed.

For the post-weaning traits, scrotal circumference (SC), weight gain from weaning to 18 months of age (WG) and muscle score (MUSC), two methods of contemporary group formation were utilized. The first (F WEMANG), considered the concatenation of the effects: farm at weaning season, year of birth, gender, weaning management group, farm at postweaning season and post-weaning management group. Furthermore, as an alternative model (R_WEMANG), the weaning management group was considered as a random effect and not included as a fixed effect in the CG. For the pre-weaning traits, birth weight (BW) and weaning weight (WW), the CG structures were similar to those of post-weaning traits, but only considering the effects corresponding to the season of each trait.

The animal distribution through the CGs for models F_WEMANG and R_WEMANG are presented in Table 1.

\begin{tabular}{|c|c|c|c|c|c|}
\hline Models & Traits & $\mathrm{N}$ & $\mathrm{N}$ CG & MIN_AN & MAX_AN \\
\hline \multirow{5}{*}{ F_WEMANG } & BW & 66,113 & 1497 & 10 & 714 \\
\hline & WW & 68,553 & 1731 & 10 & 279 \\
\hline & $\mathrm{SC}$ & 29,794 & 2926 & 10 & 279 \\
\hline & WG & 58,337 & 2726 & 10 & 275 \\
\hline & MUSC & 58,232 & 2714 & 10 & 275 \\
\hline \multirow[t]{3}{*}{ R_WEMANG } & $\mathrm{SC}$ & 29,810 & 939 & 10 & 1024 \\
\hline & WG & 58,660 & 978 & 10 & 726 \\
\hline & MUSC & 58,272 & 976 & 10 & 726 \\
\hline
\end{tabular}

$\mathrm{BW}=$ birth weight; $\mathrm{WW}=$ weaning weight; $\mathrm{SC}=$ scrotal circumference; $\mathrm{WG}=$ weight gain from weaning to 18 months of age; MUSC $=$ muscle score.

In the analysis models, the covariables considered were: animal age, Julian date of 
birth (representing the birth season period), dam age, and also effect of CG. Furthermore, the direct and maternal genetic effects, along with the permanent environment effect associated with the dam, were considered as random effects. MUSC was the only trait that did not include the maternal effect in its model because it did not show a significant effect in previous analyses. In matrix notation, all models utilized may be presented as follows:

$$
\mathrm{y}=\mathrm{X} \beta+\mathrm{Zg}+\mathrm{Mm}+\mathrm{Ww}+\mathrm{Pp}+\mathrm{e}
$$

(Equation 1)

where $y$ is an $\mathrm{N} \mathrm{x} 1$ vector of records; $\beta$ denotes the vector of fixed effects; $X$ is the matrix that associates $\beta$ with $y ; g$ is the vector of direct genetic effects; $Z$ is the matrix that associates $g$ with $y$; $m$ is the vector of maternal genetic effects; $M$ is the matrix that associates $m$ with $y ; w$ is the vector of management group at weaning effects; $W$ is the matrix that associates $w$ with $y ; p$ is the vector of permanent environmental plus non-additive genetic effects contributed by dams to records of their progeny; $P$ is the matrix that associates $p$ with $y$; and $e$ is the vector of residual effects, peculiar to observations, that are not explained by other parts of the model. All models assumed the existence of covariance between direct and maternal effects.

The methodology of mixed models was utilized to calculate the genetic parameters, using the animal model (Henderson, 1975), and considering all the traits simultaneously (multitrait analysis). The VCE 6.0 software (Groeneveld et al., 2008) was used for these analyses. Furthermore, for the males, the ratio of coincident animals between the models R_WEMANG and F_WEMANG was determined when selecting $20 \%$ of the top males for expected progeny difference (EPD), according to the method applied by Mourão (2005). The "software" used to estimate the breeding values for the calculation of EPDs was PEST2 (Groeneveld et al., 2009).

\section{RESULTS AND DISCUSSION}

The descriptive statistics and estimates of the variance components for BW, WW, SC, WG, and MUSC are presented in Tables 2 and 3, respectively.

\begin{tabular}{|c|c|c|c|c|c|}
\hline Traits & Mean & $\mathrm{CV}$ & SD & Min & Max \\
\hline $\mathrm{BW}$ & 31.43 & 12 & 3.62 & 13.00 & 58.00 \\
\hline WW & 190.09 & 14 & 27.04 & 61.00 & 317.00 \\
\hline $\mathrm{SC}$ & 27.23 & 13 & 3.42 & 17.00 & 40.00 \\
\hline WG & 114.45 & 27 & 31.39 & 0.50 & 294.54 \\
\hline MUSC & 3.68 & 25 & 0.91 & 1.00 & 6.00 \\
\hline
\end{tabular}

The inclusion of WEMANG as a random effect had a small influence on the results of (co)variance estimates of the traits studied. For all traits, the heritability coefficients and genetic correlations estimated using the two models were similar, and therefore, the utilization of R_WEMANG model does not interfere in the estimation of genetic parameters. These results are in accordance with Van Vleck (1987) and Visscher and Goddard (1993), who studied traits in dairy cattle. These authors mentioned that the inclusion of CG as random effect led to an increase in the number of daughters for each animal evaluated without affecting the prediction of estimated values. The genetic parameters are presented in Table 4, first for the model that does not include the WEMANG as a random effect and then for the model that does. 


\begin{tabular}{|c|c|c|c|c|c|c|}
\hline Model & Parameters & BW & WW & $\mathrm{SC}$ & WG & MUSC \\
\hline \multirow{5}{*}{ F_WEMANG } & $\sigma^{2}$ & 3.30 & 88.87 & 2.99 & 123.71 & 0.14 \\
\hline & $\sigma^{2^{\mathrm{g}}}$ & 0.66 & 63.56 & 0.64 & 43.42 & 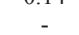 \\
\hline & $\sigma^{2}{ }^{\mathrm{m}}$ & 0.35 & 12.45 & - & - & - \\
\hline & & 5.96 & 200.87 & 3.46 & 252.55 & 0.40 \\
\hline & $\sigma_{\text {wemang }}^{2}$ & - & - & - & - & - \\
\hline \multirow{5}{*}{ R_WEMANG } & $\begin{array}{l}\text { wemang } \\
\sigma^{2}\end{array}$ & 3.30 & 88.22 & 3.03 & 127.18 & 0.14 \\
\hline & $\sigma^{2^{g}}$ & 0.67 & 64.39 & 0.64 & 43.90 & $\begin{array}{l}0.17 \\
-\end{array}$ \\
\hline & $\sigma^{2}{ }^{m}$ & 0.35 & 12.16 & $\begin{array}{l}0.07 \\
-\end{array}$ & - & - \\
\hline & $\sigma^{\sigma^{p e}}$ & 5.96 & 201.35 & 3.44 & 253.57 & 0.40 \\
\hline & $\sigma_{\text {wemang }}^{2}$ & - & - & 0.30 & 68.33 & 0.02 \\
\hline
\end{tabular}

$\sigma_{\mathrm{g}}^{2}=$ variance of direct additive genetic effects; $\sigma_{\mathrm{m}}^{2}=$ variance of maternal additive genetic effects; $\sigma_{\mathrm{pe}}^{2}=$ variance of the permanent environment effects; $\sigma_{\mathrm{e}}^{2}=$ variance of residual effects; $\sigma_{\text {wemang }}^{2}=$ variance of the weaning management group effects.

Table 4. Heritability coefficients, direct and maternal ( _ M) are presented in diagonal and genetic correlation between birth weight (BW), weaning weight (WW), scrotal circumference (SC), weight gain from weaning to 18 months of age (WG), and muscle score (MUSC) are above the diagonal, with standard errors in parentheses.

\begin{tabular}{|c|c|c|c|c|c|c|c|c|c|}
\hline & BW & WW & SC & WG & MUSC & BW_M & WW_M & SC_M & WG_M \\
\hline \multicolumn{10}{|c|}{ F_WEMANG } \\
\hline BW & $0.32(0.02)$ & $0.64(0.02)$ & $0.07(0.01)$ & $0.21(0.01)$ & $0.19(0.02)$ & $0.05(0.01)$ & $0.06(0.00)$ & $0.01(0.00)$ & $-0.20(0.02)$ \\
\hline WW & - & $0.22(0.01)$ & $0.26(0.02)$ & $0.48(0.02)$ & $0.57(0.02)$ & $0.29(0.01)$ & $0.23(0.02)$ & $-0.07(0.01)$ & $-0.32(0.02)$ \\
\hline $\mathrm{SC}$ & - & - & $0.46(0.02)$ & $0.35(0.02)$ & $0.35(0.01)$ & $0.08(0.00)$ & $0.17(0.01)$ & $-0.24(0.02)$ & $-0.23(0.02)$ \\
\hline WG & - & - & - & $0.38(0.03)$ & $0.77(0.03)$ & $0.33(0.02)$ & $0.56(0.02)$ & $0.15(0.02)$ & $-0.61(0.02)$ \\
\hline MUSC & - & _- & - & - & $0.26(0.01)$ & $0.29(0.01)$ & $0.50(0.02)$ & $0.05(0.01)$ & $-0.51(0.01)$ \\
\hline BW M & - & _- & - & - & - & $0.06(0.01)$ & $0.23(0.02)$ & $0.05(0.01)$ & $-0.10(0.01)$ \\
\hline $\mathrm{WW} \mathrm{M}$ & - & - & - & - & - & - & $0.16(0.02)$ & $0.31(0.01)$ & $-0.96(0.02)$ \\
\hline $\mathrm{SC} \overline{\mathrm{M}}$ & - & - & - & - & - & - & - & $0.10(0.01)$ & $-0.29(0.02)$ \\
\hline WG $\mathrm{M}$ & - & - & - & - & - & - & - & - & $0.13(0.02)$ \\
\hline \multicolumn{10}{|c|}{$R$ WEMANG } \\
\hline$\overline{B W}$ & $0.31(0.02)$ & $0.64(0.02)$ & $0.02(0.01)$ & $0.20(0.01)$ & $0.18(0.02)$ & $0.04(0.00)$ & $0.05(0.00)$ & $0.01(0.00)$ & $-0.19(0.02)$ \\
\hline WW & - & $0.22(0.02)$ & $0.25(0.02)$ & $0.46(0.02)$ & $0.56(0.03)$ & $0.28(0.01)$ & $0.22(0.02)$ & $-0.08(0.01)$ & $-0.32(0.02)$ \\
\hline $\mathrm{SC}$ & - & -.01 & $0.45(0.02)$ & $0.35(0.02)$ & $0.34(0.02)$ & $0.08(0.00)$ & $0.16(0.01)$ & $-0.25(0.02)$ & $-0.23(0.01)$ \\
\hline WG & - & - & -10 & $0.31(0.03)$ & $0.76(0.04)$ & $0.33(0.02)$ & $0.55(0.02)$ & $0.14(0.02)$ & $-0.62(0.03)$ \\
\hline MUSC & - & - & - & -.001 & $0.25(0.01)$ & $0.29(0.02)$ & $0.50(0.02)$ & $0.05(0.01)$ & $-0.51(0.01)$ \\
\hline BW M & - & - & - & - & - & $0.06(0.01)$ & $0.24(0.02)$ & $0.04(0.00)$ & $-0.13(0.01)$ \\
\hline WW M & - & - & - & - & - & - & $0.16(0.02)$ & $0.31(0.02)$ & $-0.96(0.02)$ \\
\hline $\mathrm{SC} \overline{\mathrm{M}}$ & - & - & - & - & - & - & - & $0.09(0.01)$ & $-0.28(0.01)$ \\
\hline $\mathrm{WG} \mathrm{M}$ & - & - & - & - & - & - & - & - & $0.11(0.02)$ \\
\hline
\end{tabular}

For BW, the results obtained for the heritability coefficients for direct additive effects were 0.32 and 0.31 for F_WEMANG and R_WEMANG models, respectively. The estimated heritability for birth weight was equivalent to the values reported by Eler et al. (2000) and Albuquerque and Meyer (2001), which ranged between 0.25 and 0.37 . The results obtained for both models were similar, demonstrating that the presence of the WEMANG effect as a random effect instead of a fixed effect in the multi-trait analysis did not influence the estimation of parameters for this trait. Heritability coefficients for WW showed the same value $(0.22)$ for both models studied, these being similar as with other studies with Nellore cattle (Horimoto et al., 2007; Boligon et al., 2008), which varied between 0.28 and 0.33. Similarly, the inclusion or exclusion of WEMANG as a random effect in multi-trait analysis for BW did not interfere in the weaning weight heritability results. 
For SC, heritability estimates considering F_WEMANG and R_WEMANG were, respectively, 0.46 and 0.45, similar to the values achieved in the literature. In Bos taurus populations, Bourdon and Brinks (1986) presented heritability results for SC in Hereford animals equal to 0.46, and Martínez-Velázquez et al. (2003) reported a mean number of 0.41 for 9 purebreds and 3 composite populations. In addition, Smith et al. (1989) obtained an average heritability estimate of 0.40 for several breeds, and for the Nellore cattle, Silva et al. (2006) and Van Melis et al. (2010) determined a heritability estimation of 0.46 and 0.42 , respectively, for the mentioned trait. For WG, heritability estimates were 0.38 for F WEMANG and 0.31 for R_WEMANG, within the range presented by Eriksson et al. (2002) (0.22 and 0.40) in European beef cattle, and of the same magnitude as obtained by Van Melis et al. (2010), with results of 0.27 and 0.25 for Nellore animals. MUSC heritability estimates for F_WEMANG and R_WEMANG were on the order of 0.26 and 0.25 , equivalent to the numbers reported by Van Melis et al. (2010), who found a heritability value of 0.23 for MUSC.

The estimates for maternal additive heritability coefficients for BW $(0.06)$ and WW (0.16) were similar to the results presented in the literature. Eler et al. (2000) obtained a value of 0.07 for BW, and Boligon et al. (2008) and Van Melis et al. (2010) presented estimates between 0.10 and 0.17 for maternal heritability of WW. The non-inclusion of maternal effects in the models for birth weight and weaning weight may result in an overestimation of the variance of direct genetic effect.

The genetic correlations estimated for both models studied, between BW and the other traits, were positive and of moderate to high magnitude, except for the correlation with SC, which was null. Therefore, selection based on production traits may lead to an increase in BW and, if not controlled, may cause an increase in dystocia. These results are in accordance with Boligon et al. (2009) who studied the correlation of BW and other productive traits.

The estimated genetic correlations between WW and the traits measured at postweaning, for both models, were high and positive, and indicated that the genes responsible for higher weaning weights are mostly the same that cause an increase in other post-weaning traits. Such results are in line with those described by Barichello et al. (2010).

Correspondingly, the correlation estimates among the evaluated post-weaning traits for both models studied were high and positive, in accordance with the findings of Pedrosa et al. (2010). Higher correlation coefficient values were observed for WG and MUSC (0.77), demonstrating a strong genetic association between these traits. The correlation results showed that the addition of WEMANG as a random effect did not interfere in the estimates between direct effects compared to the utilization of WEMANG as an effect in the contemporary group. It is expected, as shown by Van Vleck (1987), Ugarte et al. (1992) and Visscher and Goddard (1993), that the estimates of heritabilities and genetic correlations provide a satisfactory response of the true-value parameters when the random model is used, with the advantage of inclusion of a larger amount of information in the genetic evaluation.

In addition, the classification of the $20 \%$ best males for the EPDs of each trait studied was performed to identify selection errors between the different models. The ratio of coinciding animals between the models R_WEMANG and F_WEMANG was, respectively, 98, 95.5, 90.5, 91.5, and $89.5 \%$ for BW, WW, SC, WG, and MUSC. In applied terms, selection errors in which some of the best animals were not selected when F_WEMANG was utilized varied between 2 and $10.5 \%$. These selection errors occur in animals near the threshold and not for the $10 \%$ superior animals. Furthermore, this ratio would be even greater if the proportion of selected animals were closer to $50 \%$ of total. The impact of such change, for each trait, may be observed graphically in Figures 1 to 5 . 


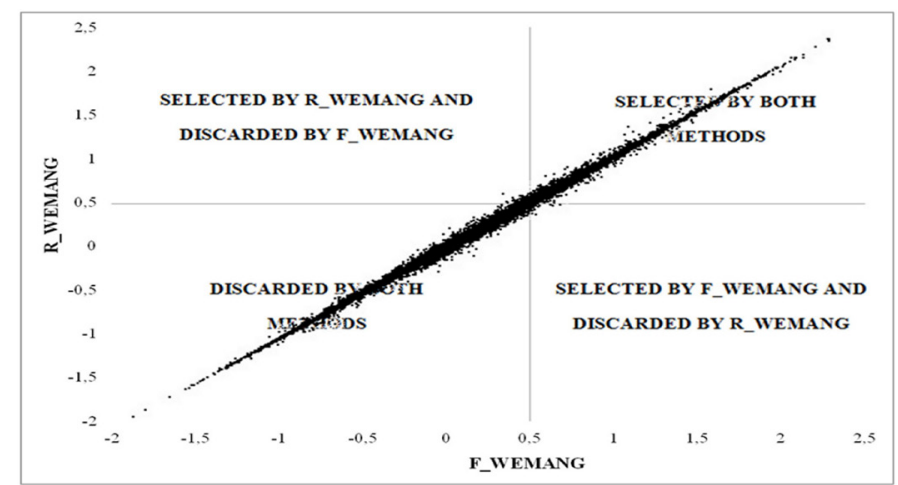

Figure 1. Dispersions of expected progeny differences (EPDs) for birth weight in quadrants defined by the truncation points of the top $20 \%$ males for R_WEMANG (EPD $\geq 0.5)$ and F_WEMANG (EPD $\geq 0.5)$ models.

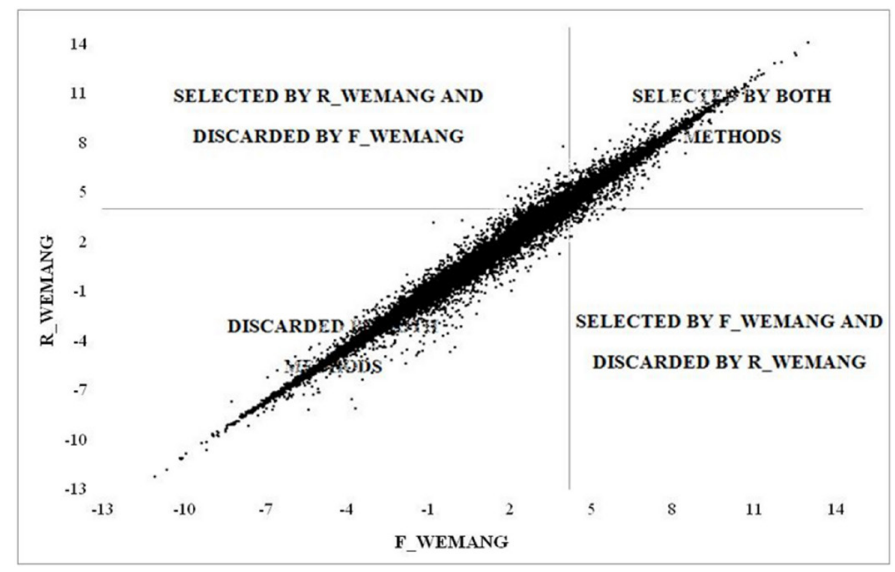

Figure 2. Dispersions of expected progeny differences (EPDs) for weaning weight in quadrants defined by the truncation points of the top $20 \%$ males for R_WEMANG (EPD $\geq 4.5)$ and F_WEMANG (EPD $\geq 4.5)$ models.

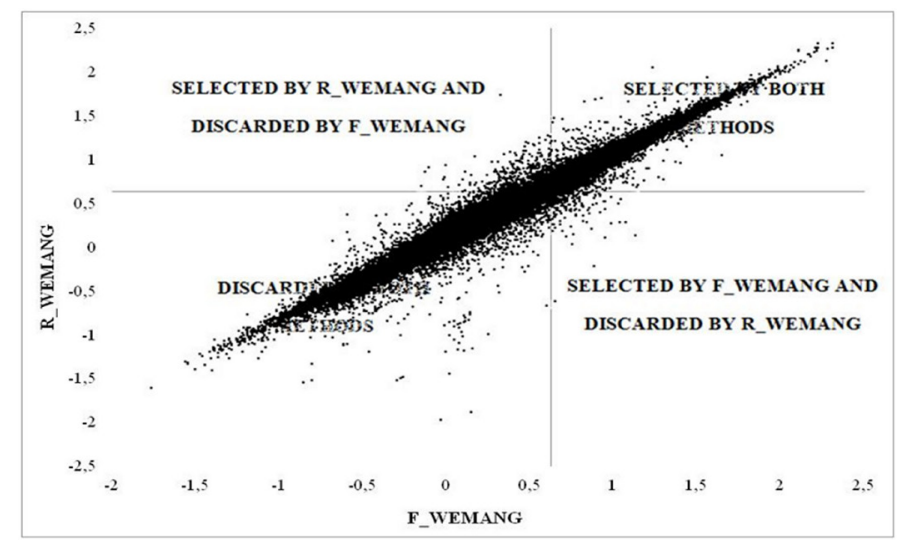

Figure 3. Dispersions of expected progeny differences (EPDs) for scrotal circumference in quadrants defined by the truncation points of the top $20 \%$ males for R_WEMANG (EPD $\geq 0.6$ ) and F_WEMANG (EPD $\geq 0.7)$ models. 


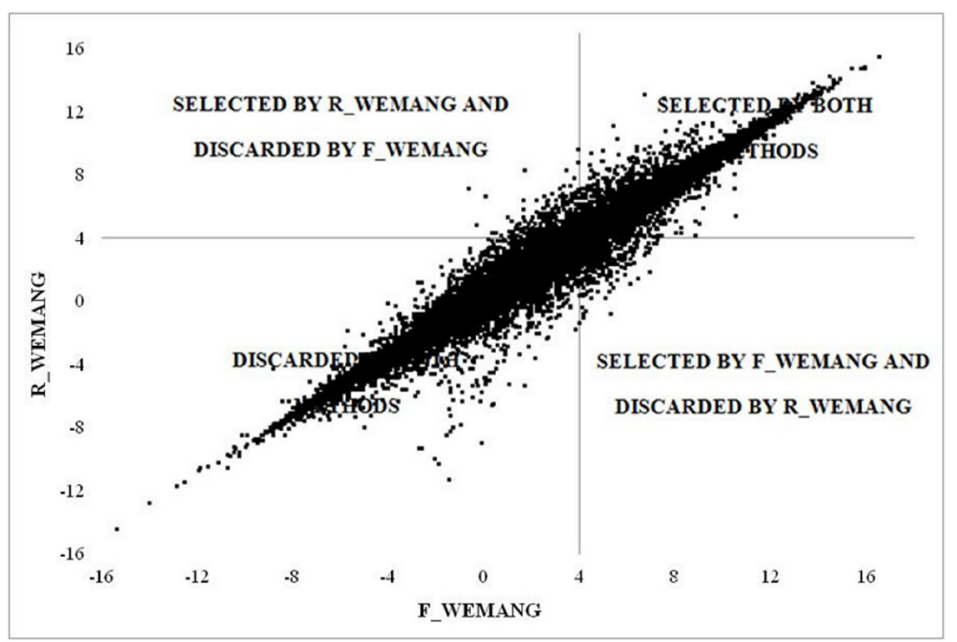

Figure 4. Dispersions of expected progeny differences (EPDs) for weight gain from weaning to 18 months of age in quadrants defined by the truncation points of the top $20 \%$ males for R_WEMANG (EPD $\geq 4.0$ ) and F_WEMANG $(\mathrm{EPD} \geq 4.0)$ models.

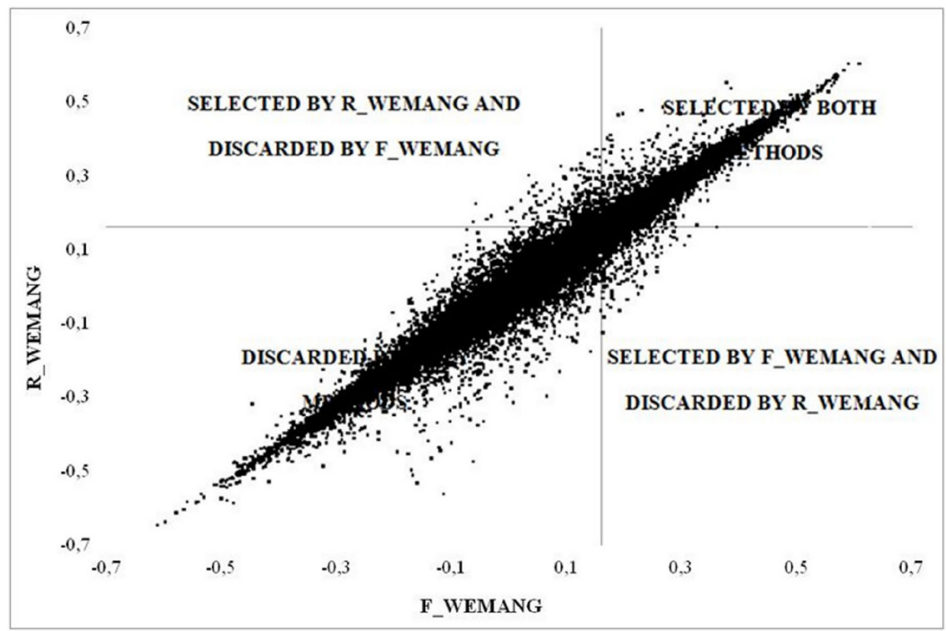

Figure 5. Dispersions of expected progeny differences (EPDs) for muscle score in quadrants defined by the truncation points of the top $20 \%$ males for R_WEMANG (EPD $\geq 0.15)$ and F_WEMANG (EPD $\geq 0.16$ ) models.

\section{CONCLUSION}

The utilization of the R_WEMANG model for a large dataset does not alter the results of heritability coefficients, demonstrating that the inclusion of the WEMANG as a random effect is an advantage, since it enables an increase in the number of animals per CG, thus avoiding the creation of CGs with small variability and, consequently, possible elimination of important animals for genetic evaluation. 


\section{ACKNOWLEDGMENTS}

Research supported by FAPESP. We are grateful to Agropecuária CFM Ltda. (Agricultural Livestock CFM Ltd.) for supplying the dataset.

\section{REFERENCES}

Albuquerque LG and Meyer K (2001). Estimates of direct and maternal genetic effects for weights from birth to 600 days of age in Nelore cattle. J. Anim. Breed. Genet. 118: 83-92.

Barichello F, Alencar MM, Torres Junior RAA and Silva LOC (2010). Heritability and correlations for weight, scrotal circumference and visual scores at weaning, in Canchim beef cattle. Pesq. Agropec. Bras. 45: 563-570.

Boligon AA, Albuquerque LG and Rorato PRN (2008). Genetic associations among weights and reproductive traits in Nellore cattle. R. Bras. Zootec. 37: 596-601.

Boligon AA, Albuquerque LG, Mercadante MEZ and Lôbo RB (2009). Heritability and correlations between weights from birth to maturity in Nellore cattle. Braz. J. Anim. Sci. 38: 2320-2326.

Bourdon RM and Brinks JS (1986). Scrotal circumference in yearling Hereford bulls: adjustment factors, heritabilities and genetic, environmental and phenotypic relationships with growth traits. J. Anim. Sci. 62: 958-967.

Eler JP, Ferraz JBS, Golden BL and Pereira E (2000). Influence of Sire x Herd interaction on the estimation of correlation between direct and maternal genetic effects in Nellore cattle. Braz. J. Anim. Sci. 29: 1642-1648.

Eriksson S, Näsholm A, Johansson K and Philipsson J (2002). Genetic analysis of post-weaning gain of Swedish beef cattle recorded under field conditions and at station performance testing. Livest. Prod. Sci. 76: 91-101.

Groeneveld E, Kovac M and Mielenz N (2008). VCE User's Guide and Reference Manual. Version 6.0. Department of Animal Science, University of Illinois, Urbana.

Groeneveld E, Kovac M and Mielenz N (2009). PEST2 User's Guide and Reference Manual. Version 2.0. Department of Animal Science, University of Illinois, Urbana.

Henderson CR (1975). Comparison of alternative sire evaluation methods. J. Anim. Sci. 41: 760-770.

Horimoto AR, Ferraz JB, Balieiro JC and Eler JP (2007). Phenotypic and genetic correlations for body structure scores (frame) with productive traits and index for CEIP classification in Nellore beef cattle. Genet. Mol. Res. 6: 188-196.

Martínez-Velázquez G, Gregory KE, Bennett GL and Van Vleck LD (2003). Genetic relationships between scrotal circumference and female reproductive traits. J. Anim. Sci. 81: 395-401.

Mourão GB (2005). Estimação de Efeitos Genéticos Aditivos Diretos e Maternos e Não Aditivos e, Predição do Desempenho de Pesos, Perímetro Escrotal e Musculosidade em uma População de Bovinos de Corte Compostos (Bos taurus x Bos indicus). PhD thesis, Faculdade de Zootecnia e Engenharia de Alimentos, Universidade de São Paulo, Pirassununga.

Pedrosa VB, Eler JP, Silva JAIIdeV, Ribeiro S, et al. (2010). Genetic parameters for mature weight and growth traits in Nellore cattle. Rev. Bras. Saúde Prod. Anim. 11: 104-113.

Pollak EJ and Quaas RL (1983). Definition of group effects in sire evaluation models. J. Dairy Sci. 66: 1503-1509.

Silva JAIIV, Formigoni IB, Eler JP and Ferraz JBS (2006). Genetic relationship among stayability, scrotal circumference and post-weaning weight in Nelore cattle. Livest. Sci. 99: 51-59.

Smith BA, Brinks JS and Richardson GV (1989). Estimation of genetic parameters among reproductive and growth traits in yearling heifers. J. Anim. Sci. 67: 2886-2891.

Ugarte E, Alenda R and Carabaño MJ (1992). Fixed or random contemporary groups in genetic evaluations. J. Dairy Sci. 75: 269-278.

Van Melis MH, Oliveira HN, Eler JP, Ferraz JB, et al. (2010). Additive genetic relationship of longevity with fertility and production traits in Nellore cattle based on bivariate models. Genet. Mol. Res. 9: 176-187.

Van Vleck LD (1987). Contemporary groups for genetic evaluations. J. Dairy Sci. 70: 2456-2464.

Vasconcelos J, Santos F, Bagnato A and Carvalheira J (2008). Effects of clustering herds with small-sized contemporary groups in dairy cattle genetic evaluations. J. Dairy Sci. 91: 377-384.

Visscher PM and Goddard ME (1993). Fixed and random contemporary groups. J. Dairy Sci. 76: 1444-1454. 